\title{
Reducing Attendance Time in LR-EPONs With Differentiated Services
}

\author{
Amr Elrasad and Basem Shihada \\ CEMSE, King Abdullah University of Science and Technology (KAUST), Saudi Arabia
}

\begin{abstract}
This work presents a novel on-the-fly void filling scheme for Long-Reach EPON called Size Controlled Batch Void Filling (SCBVF). SCBVF aims at reducing the time between consecutive bandwidth grants (attendance time) and hence reducing the average delay for delay-sensitive traffic.
\end{abstract}

\section{INTRODUCTION}

The packet delay composition of Ethernet Passive Optical Networks (EPONs) is shown in Fig.1. The average packet delay is the sum of the time between packet arrival and report message transmission $\left(W_{\text {poll }}\right)$, the time between report message transmission and the bandwidth grant allocation $\left(W_{\text {grant }}\right)$, and the time between grant start and packet transmission $\left(W_{\text {queue }}\right) . W_{\text {grant }}$ might span multiple cycles (i.e. $W_{\text {grant }}>R T T$, where $R T T$ is the round-trip-time). It can be noted that $\bar{W} \geq 1.5 \overline{R T T}$. We define $W_{\text {att }}$ to be the time between two consecutive bandwidth grants (attendance time). In Long-Reach EPONs (LR-EPONs), the reach can be up to $100 \mathrm{~km}$ which corresponds to $R T T$ of $1 \mathrm{~ms}$. Hence no packet can be transmitted less than $1 \mathrm{~ms}$ after its arrival (i.e. $W_{a t t}>1 \mathrm{~ms}$ ). This large gap between grants negatively affects the delay-sensitive traffic such as voice and multimedia traffic.

In this work, we present on-the-fly void filling scheme to help reducing $W_{a t t}$ and make better utilization of voids between successive bandwidth grants.

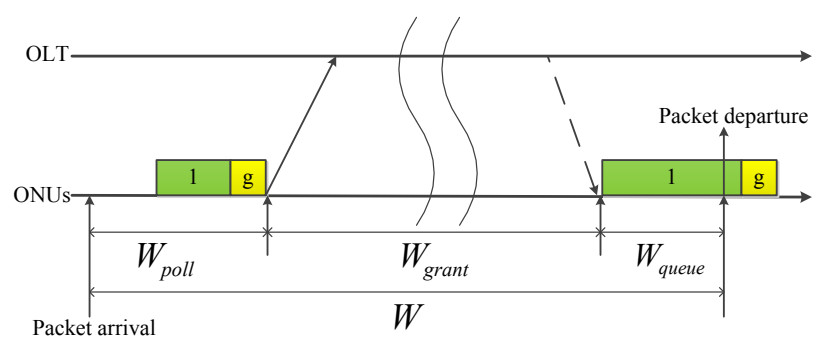

Fig. 1: Delay composition in EPON.

\section{RELATED WORK}

In [1], authors presented a request based void filling (RBVF) scheme. RBVF takes advantage of the OLT-ONU distance variance to allocate grants into voids between consecutive grants. A bandwidth grant is allocated into void if the void size is greater than or equal the bandwidth grant. In [2], authors extended RBVF to allow bandwidth grant to be divided at most $p$ chunks and hence make better void utilization. We will refer to their work as request based partial void filling (RBPVF).
The results reported in [1], [2], show that both schemes can not achieve delay lower than $1.5 \overline{R T T}$.

\section{ON-THE-FLY VOID FILLING}

On-the-fly void filling technique relies on void detection after a request based grant (RBG) is assigned by the OLT as shown in Fig.2. Upon receiving grant request from $i$ th ONU, the OLT schedules the RBG grant start time at the first free time (horizon time), $t_{h}(i, n)$. In order to detect if there is a void succeeding $i$ th ONU, the OLT compares $t_{h}(i, n)$ with the minimum start time of next node grant, $t_{s}^{\min }(i+1, n)$. $t_{s}^{\min }(i+1, n)$ equals the grant end time of cycle $n-1$ plus $R T T$ as shown in Fig.2.

Upon detecting a void, the OLT immediately fills the detected voids with void based grant(s) VBG(s). Since ONUs are polled in cyclic order, the void detection computation complexity is of $O(1)$. The size of VBGs only depends on the detected void duration. It is worth noting that during VBG, the ONU will not send bandwidth requests. In fact, bandwidth requests are only sent during RBG. On-the-fly void filling delay reduction mechanism is to enable reported packets to be partially transmitted during unutilized voids and hence reducing $W_{a t t}$. This mechanism has great effect on delay reduction for delay sensitive traffic. We propose a VBG sizing schemes to fill the detected voids namely size controlled batch void filling (SCBVF). SCBVF assigns VBGs based on

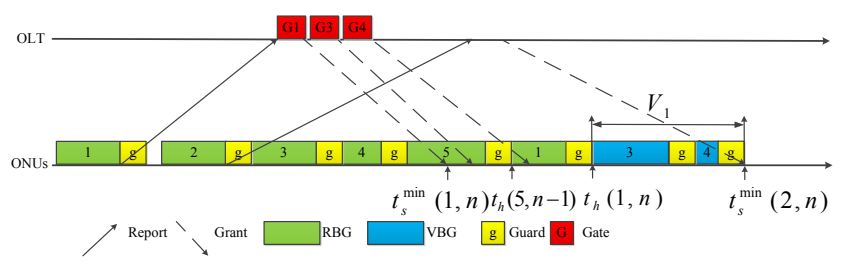

Fig. 2: Size controlled batch void filling (SCBVF).

predetermined size threshold. In SCBVF, each VBG does not exceed a certain threshold $V_{i}^{\max }$ which is set based on each ONU relative weight $w_{i}$ such that:

$$
\frac{V_{i}^{\max }}{V_{j}^{\max }}=\frac{w_{i}}{w_{j}},
$$

where $i$ and $j \in\{0,1,2, \ldots, N-1\}$ and $w_{i}$ represents the ONU relative weight according to service level agreement. SCBVF continues to allocate VBGs with the maximum void duration until a polled ONU can not be granted its maximum void 


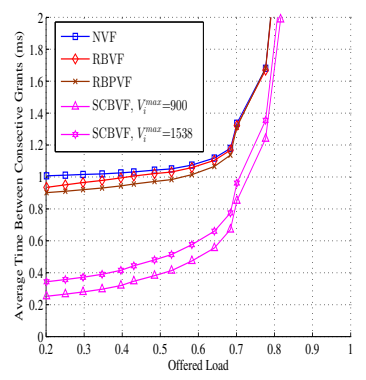

(a)

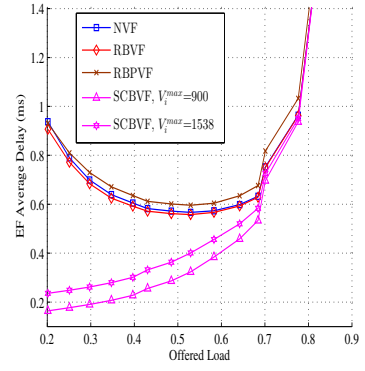

(b)

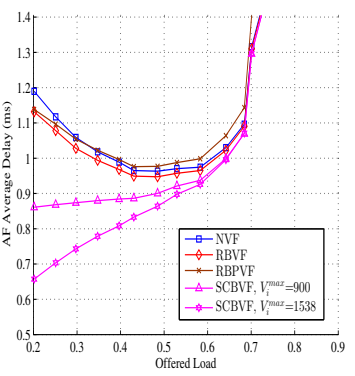

(c)

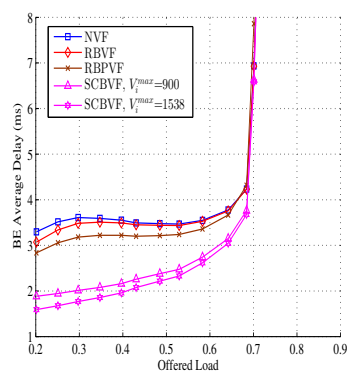

(d)

Fig. 3: (a) $\bar{W}_{\text {att }}$ (b) EF Average delay (c) AF Average delay (d) BE Average delay.

duration. In order to maximize the benefit of void filling, this ONU will be granted the remaining grant rather that leaving it unscheduled. The operation of SCBVF is shown in Fig.2. In this figure, we observe that ONU 3 has been allocated it's maximum VBG size while ONU 4 was granted the remaining part.

\section{Performance evaluation}

We consider an EPON with single OLT and 32 ONUs with $10 \mathrm{MB}$ buffer size with symmetric transmission rate of $1 \mathrm{Gbps}$. Traffic is classified to 3 classes of service: Expedited Forward (EF), Assured Forward (AF), and Best Effort (BE). EF offered load share is $20 \%$, while the rest is divided equally between $\mathrm{AF}$ and BE. EF traffic arrives with Poisson arrivals and fixed packet size of 70 bytes, while $\mathrm{AF}$ and $\mathrm{BE}$ traffic are selfsimilar with long range dependence and Hurst parameter 0.8. Maximum cycle time is set to $4 \mathrm{~ms}$ with guard time of $1 \mu \mathrm{s}$. The OLT-ONU distance os uniformly distributed between 80$100 \mathrm{~km}$. The offered load is distributed uniformly over ONUs. SCBVF is compared against non-void-filling (NVF), RBVF, and RBPVF.

Fig.3(a) shows the time between consecutive bandwidth grants for NVF, RBVF, RBPVF, and SCBVF. SCBVF achieves significant lower time period between consecutive bandwidth grants (between $0.2-0.9 \mathrm{~ms})$, while it is between $(0.9-1.2 \mathrm{~ms})$ for the other schemes. This means that SCBVF is able to utilize the voids more efficiently. The short time between grants helps ONU to flush the higher priority packets and delay sensitive traffic more frequently and thus reduces their average delay. It also helps the lower priority packets (BE) to be transmitted mainly in RBG and small portion of them during VBG.

Fig.3(b) shows EF traffic average delay performance. SCBVF achieves delay reduction ranges from 0.7 to 0.2 ms compared to NVF. It is also shown that reducing VBG size is more beneficial to EF traffic. It is clear that SCBVF $\mathrm{EF}$ performance improves with decreasing $V_{i}^{\max }$. SCBVF continues outperforming its peers for AF traffic also (Fig.3(c)). It is worth noting that increasing $V_{i}^{\max }$ helps reducing SCBVF AF average delay. This because the bigger $V_{i}^{\max }$ allows more EF packets to be transmitted within VBG(s).
Regarding BE traffic (Fig.3(d)), SCBVF reduces average delay by $\approx 1 \mathrm{~ms}$ at low load and $\approx 0.4 \mathrm{~ms}$ at high load. BE packets are rarely transmitted during VBG due to its lower priority. It makes use of the empty space at RBG slot due to more $\mathrm{EF}$ and $\mathrm{AF}$ packets transmitted during $\mathrm{VBG}(\mathrm{s})$. It is also important to highlight that NVF, RBVF, and RBPVF suffer from light load penalty phenomena [3] for all traffic classes. The light load penalty phenomena is that the average delay decreases with the load increase at lower load range. As the load becomes moderate, this phenomena disappears. Both CCBVF and SCBVF are less affected by this phenomena for relatively lower VBG size.

\section{CONCLUSION}

This paper presents a novel on-the-fly void filling approach to readuce attendance time for Long-Reach Ethernet Passive Optical Networks (LR-EPONs). We propose a VBG sizing scheme called Szie Controlled Batch Void Filling (SCBVF). For DiffServ traffic, SCBVF is able reduce the time between consecutive grants to $\approx 0.2 \mathrm{~ms}$ compared to $\approx 1 \mathrm{~ms}$ for the other schemes. This has a great impact on delay reduction for $\mathrm{EF}, \mathrm{AF}$, and $\mathrm{BE}$ traffic classes respectively. The numerical results show that the smaller the VBG size is, the lower EF delay SCBVF achieves. On the other hand, reducing VBG size increases both $\mathrm{AF}$ and $\mathrm{BE}$ traffic delay. Numerical results shows that SCBVF achieves delay reduction up to $80 \%, 45 \%$, and $52 \%$ for $\mathrm{EF}, \mathrm{AF}$, and $\mathrm{BE}$ traffic classes.

\section{REFERENCES}

[1] K. Kanonakis and I. Tomkos, "Improving the efficiency of online upstream scheduling and wavelength assignment in hybrid WDM/TDMA EPON networks," Selected Areas in Communications, IEEE Journal on, vol. 28, no. 6, pp. 838-848, Aug 2010.

[2] A. Buttaboni, M. De Andrade, and M. Tornatore, "A multi-threaded dynamic bandwidth and wavelength allocation scheme with void filling for Long-Reach WDM/TDM PONs," Lightwave Technology, Journal of, vol. 31, no. 8, pp. 1149-1157, April 2013.

[3] C. Assi, Y. Ye, S. Dixit, and M. Ali, "Dynamic bandwidth allocation for quality-of-service over Ethernet PONs," Selected Areas in Communications, IEEE Journal on, vol. 21, no. 9, pp. 1467-1477, 2003. 